\title{
The Impact of the COVID-19 Pandemic on Immunization Campaigns and Programs: A Systematic Review
}

\author{
Zohra S. Lassi ${ }^{1}$, Rabia Naseem ${ }^{2} \mathbb{}$, Rehana A. Salam ${ }^{2}$, Faareha Siddiqui ${ }^{2}$ and Jai K. Das ${ }^{2, *}$ \\ 1 Robinson Research Institute, The University of Adelaide, Adelaide, SA 5005, Australia; \\ zohra.lassi@adelaide.edu.au \\ 2 Division of Women and Child Health, The Aga Khan University, Karachi 74500, Pakistan; \\ rabianaseemm@gmail.com (R.N.); rehana.salam@aku.edu (R.A.S.); Faareha.siddiqui@gmail.com (F.S.) \\ * Correspondence: jai.das@aku.edu
}

Citation: Lassi, Z.S.; Naseem, R.; Salam, R.A.; Siddiqui, F.; Das, J.K. The Impact of the COVID-19 Pandemic on Immunization Campaigns and Programs: A Systematic Review. Int. J. Environ. Res. Public Health 2021, 18, 988. https://doi.org/10.3390/ijerph 18030988

Received: 22 October 2020

Accepted: 20 January 2021

Published: 22 January 2021

Publisher's Note: MDPI stays neutral with regard to jurisdictional claims in published maps and institutional affiliations.

Copyright: (c) 2021 by the authors. Licensee MDPI, Basel, Switzerland. This article is an open access article distributed under the terms and conditions of the Creative Commons Attribution (CC BY) license (https:// creativecommons.org/licenses/by/ $4.0 /)$.

\begin{abstract}
The COVID-19 pandemic has had an impact on health service delivery, including immunization programs, and this review assesses the impact on vaccine coverage across the globe and identifies the potential underlying factors. A systematic search strategy was employed on PubMed, Embase, MedRxiv, BioRxiv, and WHO COVID-19 databases from December 2019 till 15 September 2020. Two review authors independently assessed studies for inclusion, assessed quality, and extracted the data (PROSPERO registration \#CRD42020182363). A total of 17 observational studies were included. The findings suggest that there was a reduction in the vaccination coverage and decline in total number of vaccines administered, which led to children missing out on their vaccine doses. An approximately fourfold increase was also observed in polio cases in polio endemic countries. Factors contributing to low vaccine coverage included fear of being exposed to the virus at health care facilities, restriction on city-wide movements, shortage of workers, and diversion of resources from child health to address the pandemic. As the world re-strategizes for the post-2020 era, we should not let a crisis go to waste as they provide an opportunity to establish guidelines and allocate resources for future instances. High-quality supplementary immunization activities and catch-up programs need to be established to address gaps during the pandemic era.
\end{abstract}

Keywords: immunization; vaccination; coronavirus; COVID-19; polio

\section{Introduction}

The emergence of the novel severe acute respiratory syndrome coronavirus 2 (SARSCoV-2) led the world into a crisis of unprecedented scale and scope [1]. As of 9 December 2020, more than 68 million confirmed cases and 1566 thousand deaths have been reported across 219 countries and territories, causing morbidity, mortality and societal disruption on a global scale [2]. As governments across the world attempted to regulate the outbreak by implementing population-wide lockdowns, closing borders and halting mass gatherings, experts started worrying about the indirect health impacts. These disruptions are very likely to threaten the progress of various programs, including immunization campaigns, which have proven to be a valuable and cost-effective public health intervention to date [3]. In an attempt to mitigate the devastating impact of the COVID-19 pandemic, the World Health Organization $(\mathrm{WHO})$ issued guidelines calling for a temporary suspension of the operations of mass immunization programs across the globe [4,5]. While global vaccination programs were sliding backward even before this era [6], the related disruption is occurring at a scale larger than any since the advent of immunization programs in the 1970s. Consequences of such a falling through can be extensive, and could possibly even impact the future delivery of a COVID-19 vaccine.

According to the data collected by the WHO, the United Nations Children's Fund (UNICEF), the Global Alliance for Vaccines and Immunizations (GAVI), and the Sabin Vaccine Institute, the suspension of vaccination services in over 68 countries have put at 
least 80 million children under the age of one at risk [7,8]. In low- and middle-income countries (LMIC) with health systems already under strain, even temporary disruptions can leave a trail of devastating health, opening the door to a possible resurgence of other illnesses [9]. An analysis by GAVI estimated that a further 24 million individuals who were hitherto protected through vaccinations are now at risk, as approximately 90 mass vaccination campaigns had been postponed [7-10]. With a continued decline in vaccine coverage rates, cases of intensified outbreaks of measles, diphtheria, pertussis, and other vaccine preventable diseases (VPDs), are drawing headlines [11], and multiple cases of polio and diphtheria have been reported across Pakistan and Afghanistan [12]. Measles is rising around the globe [13-15], dengue is flaring in regions of Latin America [16] and the Amazonian region [17], and countries in Africa find themselves under immense strain as they concurrently contend with outbreaks of measles and Ebola [18]. These outbreaks are a stark reminder that even during a pandemic, public health challenges are just as important, if not more. Drawing on historical and epidemiological analyses, and data from recent modelling exercises, has underscored the importance of maintaining essential health services including immunization $[19,20]$. The WHO predicted an increase in mortality caused by malaria in endemic regions [21]. Similarly, historical evidence from the past global disease outbreaks (e.g., diphtheria in the former Soviet Union in 1990-1996), wars and infectious threats have shown that lapses in primary care access and shifting attention away from routine health services lead to an increase in morbidity and mortality [22]. As COVID-19 triggered a similar breakdown of immunization systems, future of a hard-fought struggle to prevent mortality from VPDs is at stake.

In this systematic review, we illustrate the potential impact of the COVID-19 pandemic on immunization coverage across the globe and identify the factors contributing to the disruption. The findings from this study would provide insights for the impact of pandemics on existing vaccination strategies and hence help inform policy for effective planning for future pandemics and other crises.

\section{Methods}

This systematic review aimed to assess the magnitude of the impact of the COVID-19 pandemic on existing vaccination programs, and identify the factors associated with the disruption. We adhered to the guidelines established by Preferred Reporting Items for Systematic Reviews and Meta-Analyses (PRISMA; Supplementary Table S1) [23], and the title has been registered with PROSPERO (International Prospective Register of Systematic Reviews) under the identifier \# CRD42020182363.

A search strategy was devised using appropriate, medical subject heading (MeSH), and free text terms to identify published (including pre-print) experimental, observational, and qualitative studies that have evaluated the impact of the COVID-19 pandemic on routine and/or mass immunization programs and campaigns. We also included studies exploring the factors associated with disruption in immunization programs and campaigns. Our search was designed to include all populations from both high-income countries and low- and middle-income countries impacted by COVID-19. We excluded opinion pieces, systematic reviews, commentaries, and news articles. Epidemiological studies that only identified the disruption of immunization coverage without reporting the impact on coverage and other outcomes of interest were also excluded. Studies pertaining to other coronavirus-related illnesses, such as Middle East respiratory syndrome (MERS) or SARS $\mathrm{CoV}-1$, were excluded. Multiple reports from the same region were combined in an attempt to avoid duplicate data. For our review, we used the following definitions for routine and mass immunization. Routine immunization (RI) is the process of vaccinating in a sustainable, reliable manner, before exposure to the disease and using all vaccines in the national vaccination schedule to ensure every person is fully immunized against VPDs [24]. Mass immunization or supplementary immunization activities (SIAs) complement routine coverage and act as a catalyst to boost immunization coverage in a defined geographical area and/or area having low coverage. It delivers immunization to a large group at one or 
more locations over a short interval of time and is employed during National Immunization Days conducted in LMICs as part of the Expanded Program on Immunization (EPI) [25].

The following principal sources of electronic reference libraries were searched to access the available data: PubMed, EMBASE, the WHO COVID-19 database, and Google Scholar. The websites MedRxiv (https://www.medrxiv.org), and BioRxiv (https:/ / www.biorxiv. org) were also searched for pre-print papers. We also searched the websites of selected development agencies or organizations such as GAVI, the vaccine alliance, UNICEF, and the WHO. No language restrictions were applied and the review included all studies published since December 2019. The last search date was 15 September 2020, and the search strategy is reported in Supplementary File S2. The cited references of retrieved articles and annotated bibliographies were cross-checked for more eligible studies. All studies identified were uploaded into the Covidence-Systematic Review Software for screening [26] and then independently sifted for relevance by two review authors (RN and ZSL), and discrepancies were resolved by discussion. Multiple reports of the same study were collated to ensure each study was the unit of interest in this review. Data were independently extracted from eligible studies by two authors (RN and FS) using the pre-designed extraction sheet. Information was extracted on year of publication, study design, geographical setting, sample size, type of immunization campaign (routine or mass) and its coverage, i.e., pre-COVID-19 and during COVID-19 (where reported), and factors impacting the campaigns. We planned to perform a meta-analysis, however due to the heterogeneity in the outcome reported it was not possible, and we have descriptively summarized the findings from the included studies. We have synthesized the findings from the studies included under the following outcomes:

- Impact on routine immunization (including immunization coverage; and pediatric clinic visits);

- Impact on mass immunizations or supplementary immunization activities; and

- Factors affecting immunization coverage

Methodological quality of included studies was assessed using the criteria specified by the National Heart, Lung, and Blood Institute [27].

\section{Results}

A total of 3588 studies were identified from database searches. After excluding duplicates and articles that did not meet the inclusion criteria, we obtained 130 articles with full-texts for further evaluation, where another 113 were excluded as ineligible. We included a total of 17 observational studies [28-44] (Figure 1).

\subsection{Characteristics of the Included Studies}

Of the 17 observational studies, 10 explored immunization campaigns in high income countries (HICs) $[29,30,32,36-40,42,44]$ and the remaining seven in LMICs $[28,31,33-35,41$, 43]. Of the studies from HICs, five were conducted in the United States [30,32,36,39,42], two in the United Kingdom [37,40], and one each in France [44], Italy [29], and Spain [38]. Of those from LMICs, four were from Pakistan [28,33,35,43], one study was conducted across two countries namely, Afghanistan and Pakistan [34], and one each was from South Africa [41] and Sierra Leone [31] (Table 1). 


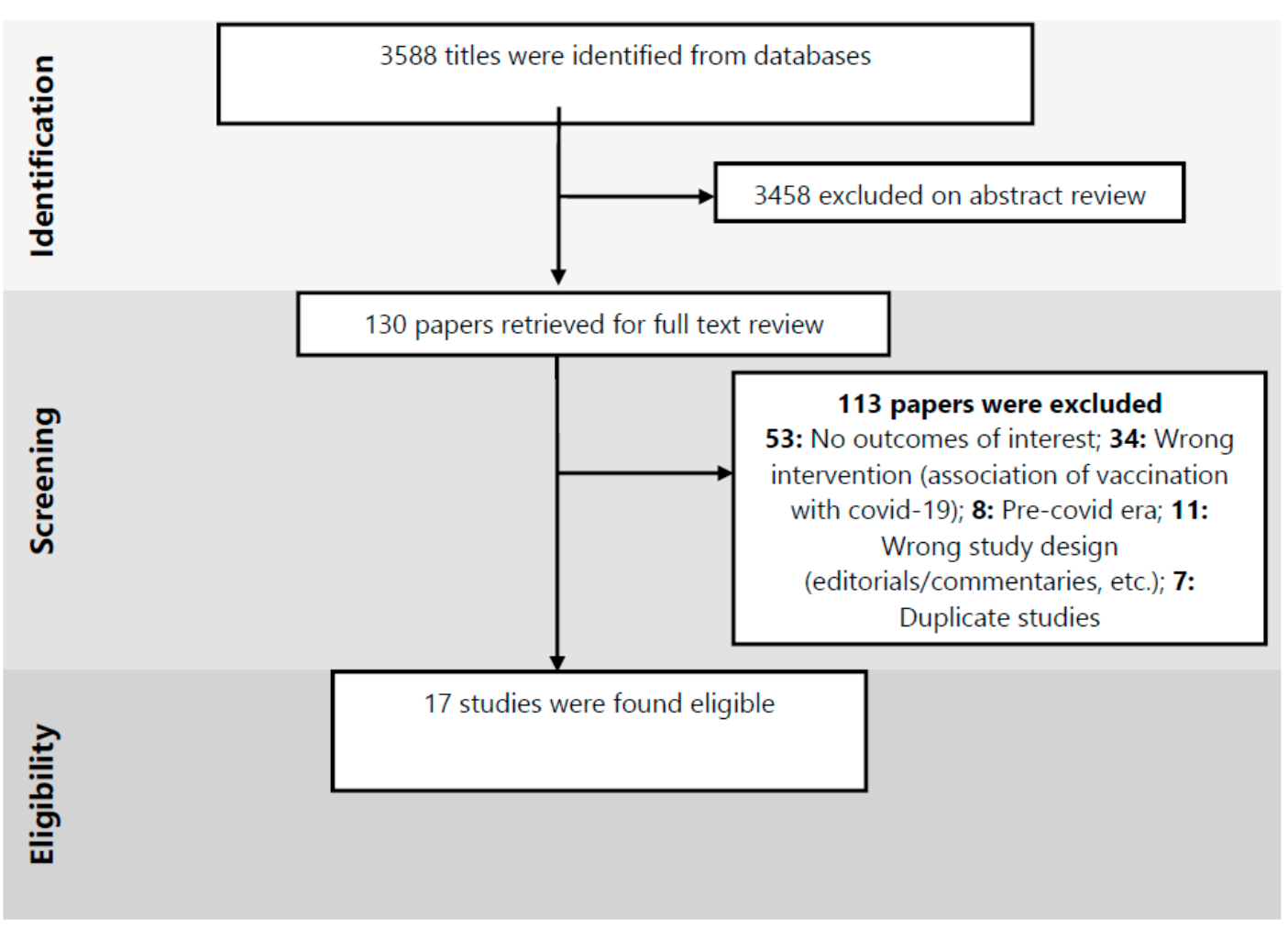

Figure 1. Preferred Reporting Items for Systematic Reviews and Meta-Analyses (PRISMA) flow diagram of study selection process.

Table 1. Characteristics of studies included.

\begin{tabular}{|c|c|c|c|c|}
\hline Author & Objective & Country/Region & Method of Retrieval & $\begin{array}{c}\text { Source of Data } \\
\text { Retrieval }\end{array}$ \\
\hline \multicolumn{5}{|l|}{ High income countries } \\
\hline Bechini 2020 [29] & $\begin{array}{l}\text { Evaluate the impact of } \\
\text { the COVID-19 } \\
\text { epidemic on pediatric } \\
\text { vaccinations } \\
\text { administered }\end{array}$ & $\begin{array}{l}\text { Tuscany, Italy, Europe } \\
\text { and Central Asia }\end{array}$ & $\begin{array}{l}\text { Members of Italian } \\
\text { Federation of } \\
\text { Pediatricians } \\
\text { participated in a } \\
\text { semi-structured } \\
\text { online survey }\end{array}$ & Project-level data \\
\hline Bramer 2020 [30] & $\begin{array}{l}\text { i. Evaluate whether } \\
\text { vaccination coverage } \\
\text { has changed during the } \\
\text { pandemic. ii. Changes } \\
\text { in vaccine doses } \\
\text { administered to } \\
\text { children and the effects } \\
\text { of those changes }\end{array}$ & $\begin{array}{l}\text { Michigan, United } \\
\text { States, North America }\end{array}$ & $\begin{array}{c}\text { Michigan Care } \\
\text { Improvement Registry }\end{array}$ & $\begin{array}{c}\text { State-level: Data } \\
\text { derived from a } \\
\text { state-level } \\
\text { immunization } \\
\text { information system, } \\
\text { i.e., Michigan Care } \\
\text { Improvement } \\
\text { Registry. }\end{array}$ \\
\hline Chanchlani 2020 [32] & $\begin{array}{l}\text { Discussed how limited } \\
\text { access to health care, } \\
\text { parental fear of seeking } \\
\text { health care, school } \\
\text { closures \& financial } \\
\text { instability as a result of } \\
\text { COVID-19 may lead to } \\
\text { increased morbidity } \\
\text { and mortality. }\end{array}$ & $\begin{array}{l}\text { United States, } \\
\text { North America }\end{array}$ & $\begin{array}{c}\text { Reports by US Vaccines } \\
\text { for Children (VFC) } \\
\text { Program }\end{array}$ & $\begin{array}{l}\text { National level-VFC } \\
\text { Program is a federally } \\
\text { funded program } \\
\text { through the CDC" }\end{array}$ \\
\hline
\end{tabular}


Table 1. Cont.

\begin{tabular}{|c|c|c|c|c|}
\hline Author & Objective & Country/Region & Method of Retrieval & $\begin{array}{c}\text { Source of Data } \\
\text { Retrieval }\end{array}$ \\
\hline $\begin{array}{l}\text { Langdon-Embry } 2020 \\
{[36]}\end{array}$ & $\begin{array}{l}\text { Weekly number of } \\
\text { routine vaccine } \\
\text { administered to } \\
\text { persons aged }<24 \\
\text { months \& } 2-18 \text { years in } \\
2020 \text { vs. } 2019\end{array}$ & $\begin{array}{l}\text { New York City, United } \\
\text { States, North America }\end{array}$ & $\begin{array}{l}\text { Citywide } \\
\text { immunization } \\
\text { registry-2.7 million } \\
\text { patients records }\end{array}$ & $\begin{array}{l}\text { State-level: Data } \\
\text { derived from a } \\
\text { state-level } \\
\text { immunization } \\
\text { information system }\end{array}$ \\
\hline McDonald 2020 [37] & $\begin{array}{l}\text { Assess the early impact } \\
\text { of COVID-19 on } \\
\text { routine childhood } \\
\text { vaccination in England } \\
\text { to } 26 \text { April } 2020 .\end{array}$ & $\begin{array}{c}\text { England \& Scotland, } \\
\text { United Kingdom; } \\
\text { Europe and } \\
\text { Central Asia }\end{array}$ & $\begin{array}{l}\text { EHR (electronic health } \\
\text { records) } \\
\text { records-electronic } \\
\text { patient records for over } \\
2600 \text { primary care } \\
\text { practices in the UK and } \\
\text { over } 35 \text { child health } \\
\text { providers }\end{array}$ & $\begin{array}{l}\text { State/national } \\
\text { level—Data derived } \\
\text { from a SystmOne } \\
\text { software system. }\end{array}$ \\
\hline Moraga-Llop 2020 [38] & $\begin{array}{c}\text { Discusses aspects of } \\
\text { recovery of vaccination } \\
\text { coverage }\end{array}$ & $\begin{array}{l}\text { Spain, Europe and } \\
\text { Central Asia }\end{array}$ & Not defined & - \\
\hline Santoli 2020 [39] & $\begin{array}{l}\text { Assess the impact of } \\
\text { the pandemic on } \\
\text { pediatric vaccination in } \\
\text { the United States. }\end{array}$ & $\begin{array}{l}\text { United States, } \\
\text { North America }\end{array}$ & $\begin{array}{c}\text { VFC Program provider } \\
\text { order data from CDC's } \\
\text { Vaccine tracking } \\
\text { System. }\end{array}$ & $\begin{array}{l}\text { National level-The } \\
\text { VFC Program and } \\
\text { Vaccine Tracking } \\
\text { System }\end{array}$ \\
\hline Saxena 2020 [40] & $\begin{array}{l}\text { Assess the impact of } \\
\text { COVID-19 on routine } \\
\text { immunizations }\end{array}$ & $\begin{array}{l}\text { England \& Scotland, } \\
\text { United Kingdom; } \\
\text { Europe and } \\
\text { Central Asia }\end{array}$ & Not defined & Not defined \\
\hline Vogt 2020 [42] & $\begin{array}{l}\text { Assess the capacity of } \\
\text { pediatric health care } \\
\text { practices to provide } \\
\text { immunization services } \\
\text { to children during the } \\
\text { covid-19 pandemic. }\end{array}$ & $\begin{array}{l}\text { United States, } \\
\text { North America }\end{array}$ & $\begin{array}{l}\text { Survey of practices } \\
\text { participating in the } \\
\text { Vaccines for Children } \\
\text { (VFC) program }\end{array}$ & Project data \\
\hline Alain Weill 2020 [44] & $\begin{array}{l}\text { Report quantifies the } \\
\text { evolution of drug use } \\
\text { prescribed in France, } \\
\text { since the start of the } \\
\text { epidemic }\end{array}$ & $\begin{array}{l}\text { France; Europe and } \\
\text { Central Asia }\end{array}$ & $\begin{array}{l}\text { Number of users } \\
\text { measured \& compared } \\
\text { to the 'expected' } \\
\text { number of } \\
\text { consumers-estimated } \\
\text { from consumption data } \\
\text { during the same weeks } \\
\text { in } 2018 \text { and } 2019\end{array}$ & $\begin{array}{c}\text { Project data; data } \\
\text { from National } \\
\text { Hospital Discharge } \\
\text { Survey } \\
\text { (NHDS)-EPI-PHARE } \\
\text { reported dispensing } \\
\text { of reimbursed drugs } \\
\text { in pharmacies since } \\
\text { the start of the } \\
\text { epidemic }\end{array}$ \\
\hline
\end{tabular}

Low- and middle-income countries

Assess the impact on

Haqqi 2020 [28] global polio eradication initiative
Pakistan, South Asia

Not defined

Not defined

\section{Understand the potential impact of COVID-19 on child}

Buonsenso 2020 [31] vaccinations in a typical poor peripheral area of Sierra Leone
Kent, Rural Western

Area, Sierra Leone; Sub Saharan Africa

\section{Cases retrospectively} collected by the center's health workers registry book
Project- level data 
Table 1. Cont.

\begin{tabular}{|c|c|c|c|c|}
\hline Author & Objective & Country/Region & Method of Retrieval & $\begin{array}{l}\text { Source of Data } \\
\text { Retrieval }\end{array}$ \\
\hline Chandir/GAVI 2020 [33] & $\begin{array}{l}\text { Understand the impact } \\
\text { of COVID-19 } \\
\text { restrictions on routine } \\
\text { immunization coverage } \\
\text { in Karachi }\end{array}$ & $\begin{array}{l}\text { Karachi, Pakistan, } \\
\text { South Asia; }\end{array}$ & $\begin{array}{l}\text { Used data from the } \\
\text { provincial Electronic } \\
\text { Immunization Registry. }\end{array}$ & Project- level data \\
\hline Chard 2020 [34] & $\begin{array}{l}\text { Summarizes progress } \\
\text { toward polio } \\
\text { eradication during } \\
1 \text { January } 2018 \text { and } \\
31 \text { March } 2020\end{array}$ & $\begin{array}{c}\text { Afghanistan \& } \\
\text { Pakistan; South Asia }\end{array}$ & $\begin{array}{c}\text { Global Polio } \\
\text { Eradication Initiative } \\
(\mathrm{GPEI})\end{array}$ & $\begin{array}{c}\text { Project } \\
\text { level—derived from } \\
\text { GPEI }\end{array}$ \\
\hline Kirmani 2020 [35] & $\begin{array}{l}\text { Study the disruption in } \\
\text { clinical activity at the } \\
\text { Children's Hospital }\end{array}$ & $\begin{array}{l}\text { Karachi, Pakistan, } \\
\text { South Asia; }\end{array}$ & $\begin{array}{l}\text { Systematically studied } \\
\text { the disruption in } \\
\text { clinical activity }\end{array}$ & Project-level data \\
\hline Siedner 2020 [41] & $\begin{array}{l}\text { Assess the impact of } \\
\text { the lockdown order in } \\
\text { response to the } \\
\text { COVID-19 epidemic in } \\
\text { South Africa }\end{array}$ & $\begin{array}{l}\text { Kwa-Zulu Natal, } \\
\text { South Africa, } \\
\text { Sub-Saharan Africa }\end{array}$ & $\begin{array}{l}\text { Data collected by the } \\
\text { Africa Health Research } \\
\text { Institute (AHRI) }\end{array}$ & $\begin{array}{c}\text { National-level } \\
\text { data-data on clinic } \\
\text { visitation at } 11 \text { public } \\
\text { health clinics }\end{array}$ \\
\hline GAVI 2020 [43] & Situation report \#14 & Pakistan, South Asia & $\begin{array}{l}\text { Expanded program on } \\
\text { immunization }\end{array}$ & Project- level data \\
\hline
\end{tabular}

Abbreviations: CDC: Centers for Disease Control, and Prevention; VFC: vaccine for children.

As indicated in Table 2, fifteen studies focused on RI campaigns and two on SIAs. Two studies reported on polio cases [28,34]; four studies assessed the coverage of Measeles, Mumps, and Rubella (MMR) vaccines during the pandemic period compared to pre-pandemic times [32,33,37,44]; while the remaining studies explored the impact on vaccination coverage as a whole. Four studies compared the number of vaccine doses administered in 2020 to the preceding years [30,31,38,43]; two studies employed the number of vaccines ordered by physicians as proxy measures to assess vaccination coverage [32,39]; four studies highlighted the decline in pediatric hospital and clinical activity during the COVID-19 period [29,35,40,41]; while three studies assessed the willingness of health care providers to provide vaccination services during the pandemic [29,31,42].

Of all of the studies included, six studies used state or national level data [32,33,35,36,40,42]. Data sources included electronic health records, reports by national vaccine programs and surveillance systems, and state registries. Eight studies reported relevant project data [29-31,34,38,41,43,44], while others did not specify the source of data [28,38,40]. The quality of the studies included using the outlined criteria is reported in Table 3. On methodological quality, studies performed well in describing the research context, however, for other quality domains, either the information was missing or not reported at all. 
Table 2. Outcomes reported in the included studies.

\begin{tabular}{|c|c|c|c|c|}
\hline Author & Time Period & Vaccine & Outcome & \\
\hline \multicolumn{5}{|c|}{ Mass immunization or supplementary immunization activities (SIAs) } \\
\hline \multicolumn{5}{|l|}{ New polio cases } \\
\hline Chard 2020 [34] & $\begin{array}{l}1 \text { January 2018-31 } \\
\text { March } 2020\end{array}$ & Polio & New polio cases & $\begin{array}{l}\text { Jan-March 2020: } 54 \text { cases- } 12 \text { in Afghanistan and } 42 \text { in Pakistan } \\
\text { Jan-March 2019: } 12 \text { cases- } 6 \text { in Afghanistan and } 6 \text { in Pakistan }\end{array}$ \\
\hline \multicolumn{5}{|l|}{$\begin{array}{l}\text { Immunization } \\
\text { coverage }\end{array}$} \\
\hline Bramer 2020 [30] & $\begin{array}{l}\text { a point in time in } \\
\text { May } 2020 \text { compared to } \\
\text { points in time in } \\
\text { May 2016-May } 2019\end{array}$ & $\begin{array}{l}\text { HepB; rotavirus (Rota); } \\
\text { Diphtheria, Tetanus, Pertussis } \\
\text { (DTaP); Haemophilus influenzae } \\
\text { type b; pneumococcal conjugate; } \\
\text { inactivated poliovirus; MMR; } \\
\text { varicella; hepatitis A }\end{array}$ & $\begin{array}{l}\text { Doses of vaccines administered } \\
\text { Percentage of children vaccinated }\end{array}$ & $\begin{array}{l}\text { Number of doses administered Jan-April } 2020 \text { vs. } 2018 \text { \& 2019: } \\
\text { children aged } \leq 18 \text { years decreased } 21.5 \% \\
\text { children aged } \leq 24 \text { months decreased } 15.5 \% \\
\text { Percentage of infants and children vaccinated: } \\
1 \text { month: } 2016-19: 82 ; 83 ; 82 ; 84,2020: 85 \\
3 \text { months: } 2016-19: 74 ; 75 ; 74 ; 65,2020: 61 \\
5 \text { months: } 2016-19: 66.6 ; 67.4 ; 67.3 ; 67.9,2020: 49.7 \\
7 \text { months: } 2016-19: 52 ; 54 ; 55 ; 54,2020: 44 \\
16 \text { months: } 2016-19: 52 ; 54 ; 54 ; 55 ; 2020: 40 \\
19 \text { months: } 2016-19: 60 ; 62 ; 60 ; 2020: 5524 \text { months: } 2016-19: 45 ; 47 \text {; } \\
49.50,2020: 43\end{array}$ \\
\hline
\end{tabular}


Table 2. Cont.

\begin{tabular}{|c|c|c|c|c|}
\hline Author & Time Period & Vaccine & Outcome & \\
\hline \multicolumn{5}{|c|}{ Mass immunization or supplementary immunization activities (SIAs) } \\
\hline $\begin{array}{l}\text { Buosenso } 2020 \\
\text { [31] }\end{array}$ & $\begin{array}{l}1 \text { March } 2019 \text { to } 26 \text { April } \\
2019 \text { v1 March } 2020 \text { to } 26 \\
\text { April } 2020\end{array}$ & $\begin{array}{l}\text { Bacillus Calmette-Guérin (BCG), } \\
\text { Oral poliovirus vaccines (OPV), } \\
\text { Pneumococcal vaccine } \\
\text { (PCV),Tetanus txoid (TT), } \\
\text { pentavalent vaccine (PENTA): } \\
\text { diphtheria, pertussis, tetanus, hep } \\
\text { b, hemophilus, TT, Intermittent } \\
\text { preventive treatment in infant }\end{array}$ & $\begin{array}{l}\text { Vaccines administered at a } \\
\text { health centre }\end{array}$ & 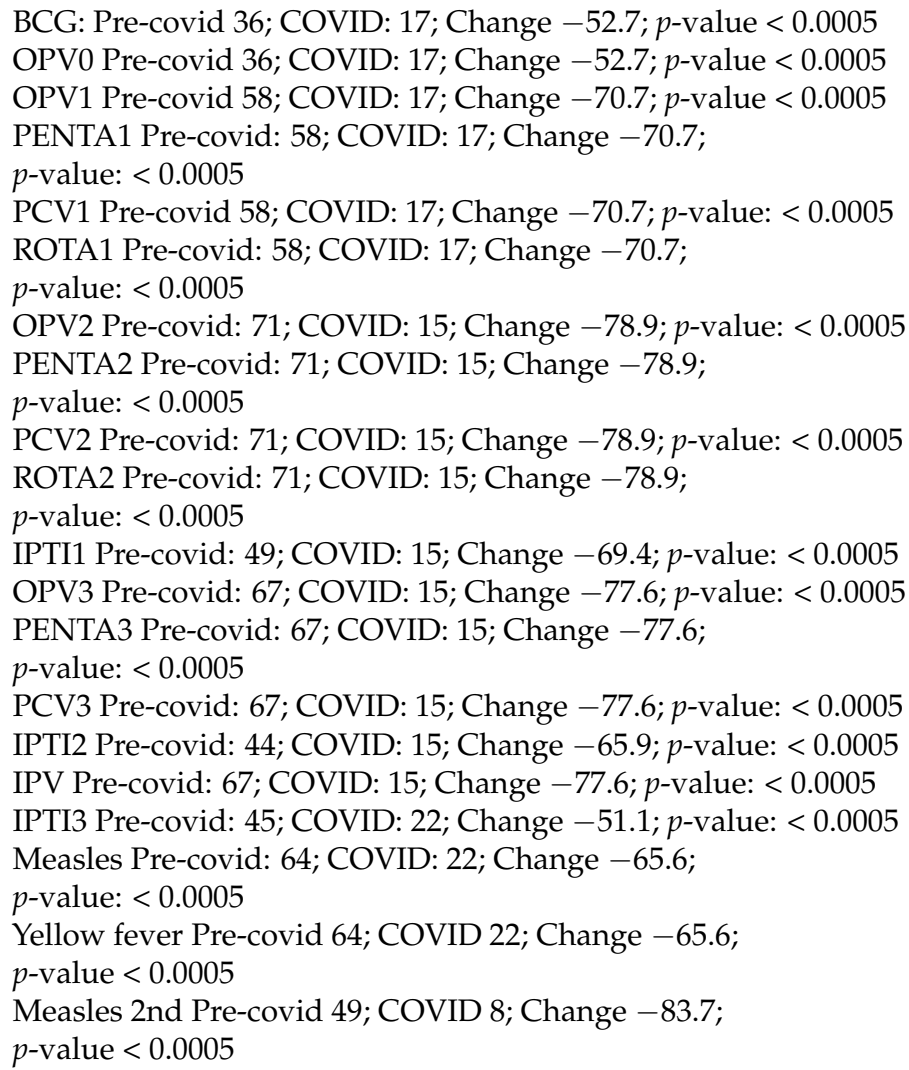 \\
\hline
\end{tabular}


Table 2. Cont.

\begin{tabular}{|c|c|c|c|c|}
\hline Author & Time Period & Vaccine & Outcome & \\
\hline $\begin{array}{l}\text { Chandir/GAVI } \\
2020[33]\end{array}$ & $\begin{array}{l}6 \text { months before the } \\
\text { lockdown (23 Sept 2019-22 } \\
\text { March 2020) } \\
\text { vs. } \\
\text { first } 6 \text { weeks of the } \\
\text { lockdown (March 23-May } \\
9,2020 \text { ) }\end{array}$ & General & $\begin{array}{l}\text { Immunization coverage } \\
\text { Visit to immunization clinic }\end{array}$ & $\begin{array}{l}\text { Daily average number of immunization } \\
6 \text { months before COVID-19 lockdown: } \\
\text { Total: } 16,649 \\
\text { Enrollments: } 32 \% \\
\text { Follow-ups: } 68 \% \\
\text { During COVID-19 lockdown: } \\
\text { Total: } 5836 \\
\text { Enrollments: } 31 \% \\
\text { Follow-ups: } 69 \% \\
\text { Immunization of children (aged 0-23 months): Baseline: } 608,832 \\
\text { children } \\
\text { Lockdown: } 92,492 \text { children } \\
\text { decrease in mean number of daily immunization visits vs baseline: } \\
\text { Lockdown (March-May 9): }-52.8 \% \text { (5184 to } 2450) \\
\text { Post-lockdown (May 10-June } 2020):-27.2 \% \text { (5184 to } 3772 \text { ). } \\
2734 \text { children per day missed routine immunization during the } \\
\text { lockdown } \\
\text { Immunization centre: } 16 \% \text { of } 321 \text { immunization centres had } \\
\text { no flow } \\
\text { reduction in immunization doses: } 88.6 \% \text { for outreach; } 38.7 \% \\
\text { for fixed } \\
\text { Vaccinators attending work: } \\
\text { Baseline: } 91.6 \% \\
\text { Lockdown: } 78.7 \%\end{array}$ \\
\hline
\end{tabular}


Table 2. Cont.

\section{Author}

Vaccine

Langdon-Embry

2020 [36]
General
Doses administerednumber of

facilities that administered at least one vaccine in 2020

Mar 1: First confirmed COVID-19 case in NYC < 24 months: 2019: 32,000; 2020: 32,0002-18 years: 2019: 19,000; 2020: 20,000 Mar 13: National emergency declared<24 months: 2019: 35,000; 2020: 31,0002-18 years: 2019: 20,000; 2020: 16,000 Mar 22: NYC on PAUSE-required New Yorkers to stay at home <24 months: 2019: 33,000; 2020: 15,0002-18 years: 2019: 21,000; 2020: 3000 April 5-11:<24 months: 62\% decrease: 2019: 33,000; 2020: 13,0002-18 years: $96 \%$ decrease: 2019: 24,000; 2020: 1000 facilities

administering at least one vaccine, $<24$ months: $46 \%$ decrease, from 900 in 2019 to 488 in 2020 facilities administering at least one vaccine, 2-18 years: decreased $78 \%$, from 1238 in 2019 to 275 in 2020 April 19-25: number of new COVID-19 cases declined $<24$ months: 2019: 27,000; 2020: 20,0002-18 years: 2019: 25,000; 2020 : 3000 June 21-27: facilities administering at least one vaccine, $<24$ months:increased $69 \%$ from the lowest point to 825 Vaccines administered to persons $2-18$ years:35\% fewer vaccines $(17,947$ doses versus 27,405).

1. Hexavalent: i. 2019: 69,568; ii. 2020: 67,116,

Percentage change: $-3.5(-3.7$ to -3.4$)$

2. MMR: i. 2019: 65, 341; ii. 2020: 61, 832

Percentage change: -3.7 ( -3.8 to -3.6$)$

Week 1-9: prior to social distancing

Hexavalent: $-5.8 \%(95 \% \mathrm{CI}-6.0$ to $-5.5 \%)$

\begin{tabular}{|c|c|c|}
\hline $\begin{array}{l}\text { McDonald } 2020 \\
{[37]}\end{array}$ & $\begin{array}{l}\text { first } 17 \text { weeks of } 2019 \text { and } \\
\text { 2020: }\end{array}$ & $\begin{array}{l}\text { i. Hexavalent: DTaP, polio, Hib } \\
\text { and Hep B } \\
\text { ii. MMR }\end{array}$ \\
\hline
\end{tabular}

MMR: $-1.0 \%(95 \%$ CI -1.1 to $-0.9 \%)$

Hexavalent: $4.4 \%(95 \% \mathrm{CI}-4.8$ to $-4.0 \%)$

MMR: $-7.2 \%$ (95\% CI -7.7 to $-6.7 \%)$

Week 13: Strict social distancing introduced

Hexavalent: $-5.1 \%(-5.9$ to -4.4$)$

MMR: -24.2 ( -25.9 to -22.5$)$

Week 13-15:

Hexavalent: $-6.7 \%$ (95\% CI -7.1 to $-6.2 \%)$

MMR: $-19.8 \%(95 \%$ CI -20.7 to $-18.9 \%)$ 
Table 2. Cont.

$\begin{array}{llll}\text { Author } & \text { Time Period } & \text { Vaccine } & \text { Outcome }\end{array}$

$\begin{array}{llll}\text { Moraga-Llop } & \text { COVID-19 era } & \text { Hexavalent, pneumococcus, } \\ 2020[38] & \text { vs. } & \text { meningococcus, MMR, Varicella, } & \text { Immunization coverage } \\ & \text { Pre-COVID-19 } & \text { Men B, Rota, Tdap }\end{array}$

meningococcus, MMR, Varicella, Immunization coverage

and $60 \%$ Reduction in vaccines administered (March 2020 vs. 2019)

2 \& 4 mths- hexavalent, pneumococcus \& meningococcus $C$.

$8-13 \%$

11-month booster doses -hexavalent and pneumococcus: $15 \%$

12-months -MMR \& meningococcus ACWY: $12 \%$

15 months- varicella: $20 \%$

Rota virus: $19 \%$

Men B: $40 \%$

pertussis vaccination in pregnant women was not affected

$(-0.6 \%)$

decrease more evident for vaccines not funded:

Valencian:

1st dose of Men B: $-68.4 \%$

Andalusia:

total doses of Men B: $-39 \%$

Rotavirus: $-18 \%$

decrease accentuated with age;

Valencian Community:

tetanus \& diphtheria ( $>64$ years of age): $-67.5 \%$.

private pediatrics did not register the same effects

$\begin{array}{llll} & \text { Mar-April } 2019 & \begin{array}{l}\text { Pentavalent 3, Oral polio vaccine } \\ \text { type 3 (OPV3), Measles, Bacillus }\end{array} & \text { Immunization coverage } \\ \text { vs. } & \text { Calmette-Guérin (BCG) } & \end{array}$

$\%$ target children covered:

Pentavalent 2019: 89, 2020: 40

Oral polio vaccine (OPV) 2019: 88, 2020: 62

Measles 2019: 84, 2020: 61

BCG 2019: 88, 2020: 64 
Table 2. Cont.

$\begin{array}{llll}\text { Author } & \text { Time Period } & \text { Vaccine } & \text { Outcome }\end{array}$

Anti- human papilloma virus

(HPV)

$\begin{array}{lll} & \text { Lockdown: March 16-May } & \text { Combined Penta/Hexavalent } \\ \text { vaccines for infants: Hæmophilu }\end{array}$

[44] 10, 2020Post-lockdown:

May 11-17, 2020

influenzæ B, pertussis,

poliomyelitis, tetanus, hep B

MMR

Anti-tetanus (excluding infants)
March 2-8: HPV: -3.6; Comb: -0.4; MMR: -13.6; Tetanus: +0.3 March 9-15: HPV: -6.8; Comb: -2.5 ; MMR: -12.3 ; Tetanus: -6.5 March 16-22: HPV: -21.9; Comb: -3.6; MMR: -28.5; Tetanus:

-26.2 March 23-29: HPV: -67.4; Comb: -23; MMR: -49; Tetanus:

-64.9 March 30-April 5: HPV: -78.1; Comb: -28.9; MMR: -50.8;

Tetanus: -77.3 April 6-12: HPV: -74.4; Comb: -21.3; MMR:

Drugs prescribed since the start of $\quad-50.8$; Tetanus: -76.5 April 13-19: HPV: -69.6 ; Comb: -18.7 ;

the epidemic

MMR: -46.9; Tetanus: -72.9 April 20-26: HPV: -45.6 ; Comb:

+ 6.1; MMR: -21.7; Tetanus: -55.2 April 30-May 3: HPV: -45.6;

Comb: + 6.1; MMR: -21.7; Tetanus: -55.2 May 4-10: HPV: -43.2;

Comb: -5.6 ; MMR: -15.7 ; Tetanus: -48.4 Difference: HPV:

-89,508; Comb: -44,171; MMR: -123,966; Tetanus: $-446,580$

Post-lockdown May 11-17: HPV: -34.4; Comb: -6.4; MMR: -4.7;

Tetanus: $-37,9$

Vaccine orders

Chanchlani 2020

[32]

6 Jan.-19 Apr. 2020, v. 2019 General, MMR, diptheria

decrease in orders

Decrease in vaccination: Apr. 5, 2020 vs Feb 16 (pre-coronavirus):

1. $50 \%$ for MMR

2. $42 \%$ for diphtheria and whooping cough

measles-containing vaccine administered: $21.5 \%$ decline (Mar,

2020)Children $<24$ months:March 9th (before US national

emergency): 2000 March 16th (After US national emergency): 1100

Children $>24$ months through 18 years: March 9th (before US

national emergency): 2250 March 16th (After US national

influenza; MMR; Meningococcal; decrease in vaccine orders in

Pneumococcal 13-\& 23 valent; IPV; period 2, compared to period 1

Rota; Tdap; Var

emergency): 500 difference in doses of vaccines ordered by health care providers:non-influenza vaccines Jan 20th (first U.S.

COVID-19 case reported): $-500,000 \mathrm{Apr} 13 \mathrm{th}:-3,000,000$

measles-containing vaccinesJan 20th: $-100,000$ Apr 13th: $-400,000$ 
Table 2. Cont.

\begin{tabular}{|c|c|c|c|c|}
\hline Author & Time Period & Vaccine & Outcome & \\
\hline \multicolumn{5}{|c|}{ Health care providers providing immunization services during the pandemic } \\
\hline Bechini 2020 [29] & $\begin{array}{l}\text { Lockdown: } 11 \text { March } 11-4 \\
\text { May } 2020\end{array}$ & Not identified & $\begin{array}{l}\text { Activities \& behavior of } \\
\text { pediatricians Visits to clinic }\end{array}$ & $\begin{array}{l}\text { 1) } 208(93.3 \%) \text { pediatricians continued to vaccinate } \\
66(31.7 \%) \text { : reduction in compliance to mandatory vaccines } \\
\text { (hexavalent and MMRV) } \\
88(42.3 \%) \text { : reduction in compliance to non-mandatory vaccines } \\
37(17.8 \%) \text { administrated only the first doses of the vaccines } \\
82.2 \%) \text { reported administering all the scheduled vaccine doses. } \\
\text { 2) } 15(7 \%) \text { had suspended vaccinations } 98.2 \% \text { - pediatricians } \\
\text { reported a general decline in outpatient visits during the } \\
\text { COVID-19 epidemic period }\end{array}$ \\
\hline Vogt 2020 [42] & 12-20 May 2020 & Not identified & Practices providing immunization & $\begin{array}{l}\text { Of } 1933 \text { practices: open: } 1727(89.8 \%) \text { providing immunization } \\
\text { services: } 1397(81.1 \%)\end{array}$ \\
\hline \multicolumn{5}{|l|}{ Visits to clinic } \\
\hline Bechini 2020 [29] & $\begin{array}{l}\text { Lockdown: } 11 \text { March-4 } \\
\text { May 2020v pre-COVID era }\end{array}$ & Not identified & Visits to clinic & $\begin{array}{l}(98.2 \%) \text { reported a general decline in outpatient pediatric visits } \\
\text { during the COVID- } 19 \text { epidemic period } 65.8 \% \text { reported a more than } \\
60 \% \text { reduction in comparison with the situation before the } \\
\text { COVID-19 pandemic. }\end{array}$ \\
\hline Kirmani 2020 [35] & $\begin{array}{l}\text { February } 2020 \text { to } \\
\text { post-lockdown }\end{array}$ & Not identified & Visits to clinic & $\begin{array}{l}\text { Week } 7 \text { (pre-COVID): } 2600 \text { Week } 8 \text { (26 Feb, First case of COVID } \\
\text { reported): 2200Week } 11 \text { (21 March, Sindh Lockdown): 1800Week } \\
\text { 13: 400Week 15: 1300Week 17: 1200Week 19: } 1300\end{array}$ \\
\hline Saxena 2020 [40] & - & Not identified & $\begin{array}{l}\text { Visits to clinic;Parental } \\
\text { concern;Vaccine coverage }\end{array}$ & $\begin{array}{l}\text { Children Visits to ED: fell by over } 90 \% \text { during April } 2020 \\
\text { Parents expressed concerns about overburdening the NHS and } \\
\text { fear of exposure to covid- } 19 \text { when attending for vaccination. } \\
60 \% \text { of } 752 \text { health visitors surveyed in May } 2020 \text { reported families } \\
\text { who had considered cancelling or postponing their } \\
\text { child's vaccinations }\end{array}$ \\
\hline
\end{tabular}


Table 2. Cont

\begin{tabular}{|c|c|c|c|c|}
\hline Author & Time Period & Vaccine & Outcome & \\
\hline Siedner 2020 [41] & $\begin{array}{l}\text { Pre lockdown: } 60 \text { days } \\
\text { prior to lockdown } \\
\text { Lockdown: } 27 \text { th } \\
\text { March-30th April' 20: } \\
\text { Post lockdown: } \\
35 \text { days after the } \\
\text { lockdown. }\end{array}$ & Not identified & Visits to clinic & $\begin{array}{l}\text { overall clinic visits during lockdown: }-0.2,(95 \% \mathrm{CI}-3.4,3.1) \\
\text { visits/day/child-health clinic: pre-lockdown period: } 11.8(8.4, \\
15.1) \\
\text { lockdown period: } 4.5 \text { mean change: }-7.2(95 \% \mathrm{Cl}-9.2,-5.3) \text {; } \\
p \text {-value: }<0.001 \\
\text { mean change post-lockdown: }+1.1(0.5,1.7) ; p \text {-value: } \\
0.001 \text { Age-stratified: Total visits Jan-Apr. } 2020: 6194 \\
<1 \text { year: } 4270(68.9 \%) \\
1-5 \text { years: } 1786(28.8 \%) \\
6-19 \text { years: } 103(1.7 \%) \\
20-45 \text { years: } 29(0.5 \%) \\
>45 \text { years: } 6(0.1 \%) \text { children under } 1 \text { : mean decrease of }-5.3 \text { visits, } \\
95 \% \mathrm{CI}-7.1,-3.61-5 \text { years: mean decrease of }-5.5 \text { visits, } 95 \% \mathrm{CI} \\
-6.8,-4.2\end{array}$ \\
\hline
\end{tabular}


Table 3. Quality of included studies.

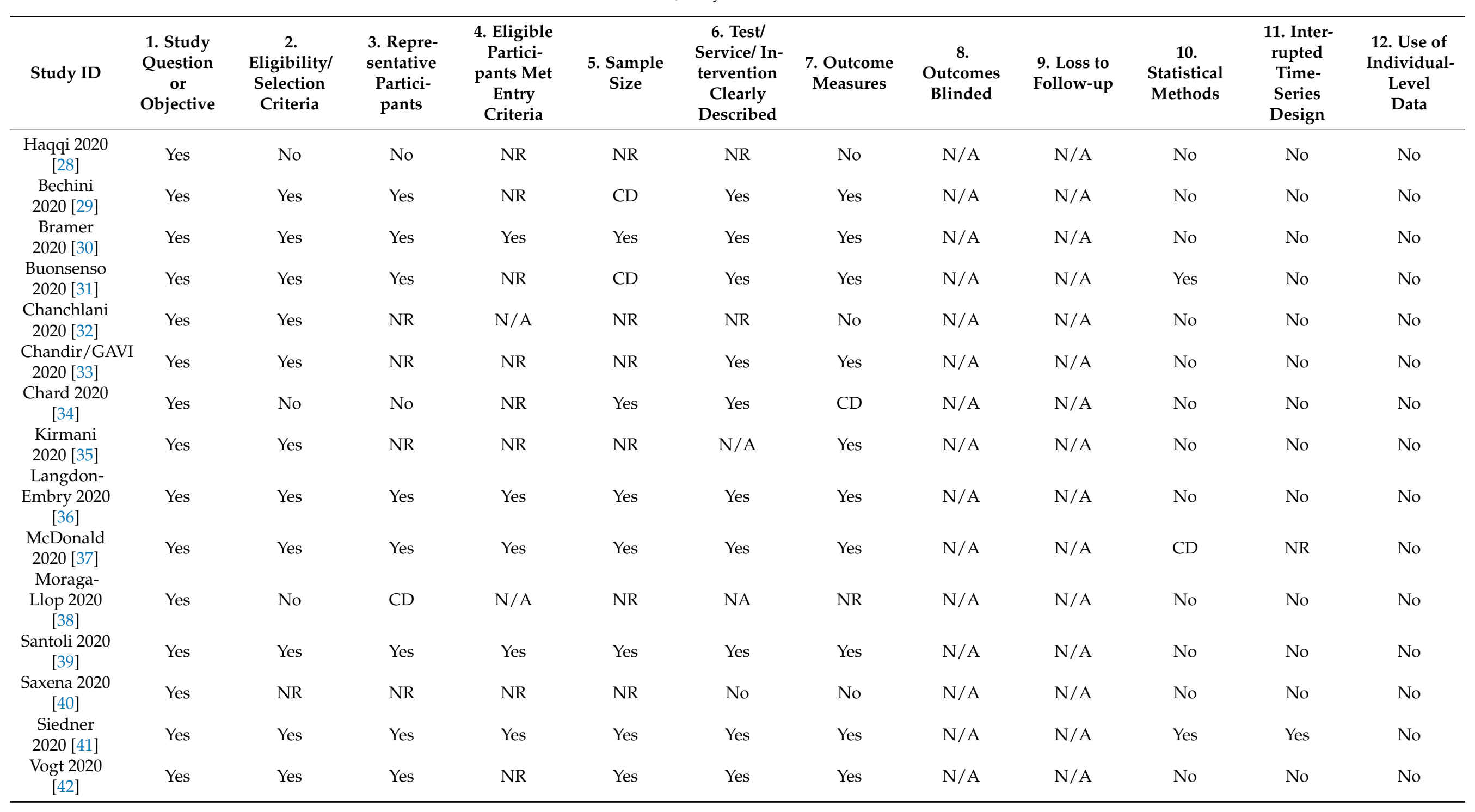




\subsection{Impact on Routine Immunization Immunization Coverage}

A total of 12 studies analyzed the impact on immunization programs during COVID19 [29-33,36-39,42-44]. Studies assessed immunization coverage during COVID-19 compared to the preceding years. The EPI-PHARE report from France contrasted the number of drugs delivered during the COVID-19 period (from 16 March to 10 May 2020) with the same weeks in 2018 and 2019. Over the course of the pandemic, vaccines delivered for infants fell by almost a quarter ( $-28.9 \%$ ) and deliveries of MMR and human papilloma virus (HPV) vaccines dropped more than half $(-50.8 \%$ and $-78.1 \%$, respectively) [44]. The Spanish Association of Pediatrics, similarly, recounted a decline in vaccination coverage ranging from 5\% to $60 \%$, in March 2020 compared to the monthly average for the period of January 2019 to February 2020 [38].

In England, the observed change in vaccinations counts in 2020 compared to 2019 varied over the course of the COVID-19 outbreak [37]. A decline in doses administered during the first 17 weeks of 2020 compared to 2019 was observed for both MMR (3.7\% lower, $95 \% \mathrm{Cl}(-3.8$ to -3.6$))$ and hexavalent $(3.5 \%$ lower, $95 \% \mathrm{Cl}(-3.7$ to -3.4$))$ vaccines. The effect was largely felt the same weeks strict social distancing measures were implemented (Week 13, 20 March 2020); following which MMR doses sharply decreased to a low point of $-24.2 \%$ from $14.7 \%$ in week 12 ). Interestingly, the decline in doses of hexavalent vaccines did not accentuate with introduction of social distancing measures (week 13: $-5.1 \%$ ).

Studies conducted in the USA outlined a substantial impact on immunization across the country (Table 2). Chanchalani et al. [32] and Santoli et al. [39] highlighted a notable decline (approximately 75\%) in vaccines ordered by doctors since January when the first person with COVID-19 was identified. Data analyzed for this subsection was provided by the vaccines for children (VFC) program provider order data. The steep decline in vaccination rates was notable for both non-influenza childhood (42\%) and measles containing vaccine $(50 \%)$. Consistent with reports of decreased vaccine counts across the country, data from state registries of Michigan and New York observed declines in vaccine doses to a similar degree; Michigan reported an approximately 18.5\% drop during January-April 2020 [30] while the New York registry reported an almost 50\% drop during COVID-19 (1 March to 27 June 2020), compared to the pre-COVID-19 era (years 2018-2019) [36]. Comparable to other countries, the largest decrease in vaccine coverage corresponded to the increase in severity of COVID-19 and the growing strictness of the lockdown, with rates of vaccination coverage improving as the course of the pandemic lightened, this impact was not just limited to HICs. Similar impact was observed in a rural area of Sierra Leone [31]. A retrospective analysis of a health facility during 1 March-26 April 2020 compared to the same period last year (1 March-26 April 2019) reported that, despite the COVID-19 pandemic, the health care center continued to function and offer their services, but the center reported a decline in children visiting the clinic for vaccinations of $57 \%$. In an observational study based in Karachi, Pakistan, Chandir et al. underscored the inequity in vaccination coverage [33]. Data of a registry employed to track vaccination status was analyzed during baseline (4 Feb 2020, to 22 Mar 2020) and compared to the COVID-19 lockdown (23 Mar 2020 to 9 May 2020) and post COVID-19 lockdown (10 May 2020 to 6 Jun 2020) period. A geographic analysis illustrated considerable spatial heterogeneity. Slum and densely-packed squatter settlements—high-risk regions with historically low immunization coverage-were the worst affected. GAVI, the vaccine alliance, in their fortnightly situation report presented a similar picture across Pakistan [43]. With outreach sessions suspended, coverage dropped by more than $50 \%$ for all vaccines. Campaigns were further impacted after several polio workers contracted COVID-19 and as an inadvertent consequence of lockdown and flight disruptions, large parts of the country also reported running out of routine vaccines.

While some studies reported age distribution for patients, no specific pattern or trend was apparent. Bramer et al. observed a decline in all milestone age cohorts, except for birthdose hepatitis B coverage which is customarily administered in hospitals [30]. The five- 
month age cohort observed a decline in vaccination status from approximately two-thirds (67.3\%) of children during 2016 to 2019 to fewer than half (49.7\%) in May 2020. In Karachi, the six-week old age group suffered the greatest drop $(60 \%)$ in immunization coverage, a decline that could eventually lead to an increase in number of zero-dose children [33]. In contrast, Santoli et al. observed a less prominent decline in measles containing vaccines among children aged $\leq 24$ months than among older children [39], and another study reported practices prioritizing offering vaccination to infants and toddlers [42]. This could suggest that system-level strategies to prioritize child care and immunization for the younger age group are robust.

Visits to clinic: Five studies assessed the disruption of pediatric clinical and hospital activities [29,33,35,40,41]. A pattern of overall decline was observed (Table 2). In South Africa, health visits declined by over $50 \%$ immediately after the lockdown (11.8 to 4.5 visits/day/clinic) was implemented, but partially bounced back post-lockdown (+1.1 visit/clinic/day) [41]. Likewise, Kirmani et al. recounted a sharp drop (1800 to 400 ) in the number of clinic visits the same week a strict lockdown was announced [35]. Parents reported fear of possible COVID-19 exposure to their children at health care facilities ultimately leading to the decline, and almost $60 \%$ of the parents had cancelled their child's appointment [40]. In another study, almost all (98.2\%) of the pediatricians surveyed reported a general decline in outpatient visits. Additionally, one study reported a decline $(53 \%)$ in the mean number of daily immunization clinic visits during the lockdown compared to 6 months before COVID-19 [33]. A survey of health care practices underscored their willingness to operate and provide services during the lockdown [42]. Likewise, a study of Tuscan pediatricians reported over $90 \%$ doctors continued to vaccinate during the period [29].

\subsection{Impact on Mass Immunization or Supplementary Immunization Activities (SIAs)}

Two included studies highlighted the breakdown of polio campaigns [28,34] (Table 2) since late March 2020, a standstill in immunization was observed amid the COVID-19 outbreak. This outbreak has coincided with growing cases of polio in Afghanistan and Pakistan. As of June 19, 2020, 54 polio cases were reported this year-an approximate fourfold increase from 12 cases in 2019 [34]. Of these, 78\% were detected in Pakistan and 22\% in Afghanistan. A closer inspection reveals that polio cases were already running high — 40 cases in Jan 2020 vs. 9 in Jan 2019—even before Global Polio Eradication Initiative (GPEI) suspended SIAs in March 2020 [34].

\subsection{Factors Affecting Immunization Coverage}

Nine studies reported factors affecting immunization coverage. The disruptions to immunization services were severe and were due to an interplay of multiple factors. Even when services were offered, people were concerned about being exposed to COVID-19 at health care facilities, fearing contacting the virus in waiting rooms and attempting to avoid contact with individuals with COVID-19 [29,31,38]. Additionally, they were reluctant to leave their homes and faced transport interruptions and restrictions on movements secondary to city-wide lockdowns [32]. Concerns of overburdening the already overstretched health care system also played a role [40]. In some regions, health workers were also unavailable because of restrictions on travel, redeployment to COVID-19 response activities, as well as the lack of protective equipment [33]. Furthermore, shuttering of educational facilities translated into a serious disruption in RI services that were previously delivered in school settings $[28,37,38,45]$. Thus, it appears that the COVID-19 pandemic and resultant chaos created a climate of fear and contributed to the unprecedented scale of disruptions of delivery and uptake of immunization services.

\section{Discussion}

Findings from this review show that the total number of vaccinations administered across all study regions began to decline precipitously during the COVID-19 pandemic. This drop in vaccinations administered coincides with both the WHO declaration describing 
the COVID-19 outbreak identified in Wuhan, China as a pandemic on March 11, 2020, and implementation of full national lockdowns, i.e., stay-at-home orders for regions or entire countries. As hospitals brace for an onslaught of critically ill patients and routine services worldwide are disrupted, health experts have had to shelve immunization campaigns for COVID-19.

COVID-19 has affected virtually all countries in the world, regardless of their economic power, but represents a particular threat for LMIC's for battling the pandemic alongside pre-existing challenges, including efforts to control VPDs and the capacity of health systems to manage the additional burden of COVID-19. Whilst the risk of COVID-19-associated mortality for children has so far been low and trivial in both LMICs and HICs [46,47], the children in LMICs are more likely to be disproportionately affected for several reasons [48]. Health systems in LMICs are under-resourced and weaker, and risk factors for poor health outcomes, such as malnutrition, poor sanitation, nutritional anemia, or human immunodeficiency virus (HIV) exposure or infection, are overwhelmingly more prevalent in LMICs than HICs $[49,50]$. Moreover, more than $90 \%$ of schools across the world faced closure, and education was interrupted due to the pandemic [51]. This further worsened inequalities in seeking education as many children in LMICs lack resources to adopt alternate arrangements [52]. With the risk of contracting communicable disease heavily skewed towards LMICs [53] and existing challenges compounded by the COVID-19 crisis, the long-term impact as a result of vaccine disruption would be severe in LMICs. Abbas et al. [54] weighed out the risks and benefits of reopening immunization clinics in the midst of the COVID-19 pandemic for LMICs in Africa. For every death attributable to SARSCoV-2 infections acquired during RI clinic visits, 84 deaths from VPDs could be prevented. Hereby, outweighing the excess risk of COVID-19 deaths associated with vaccination clinic visits. The analyses by Zar et al. [9] provided further insight into the global health inequity. Reiterating that solutions for COVID-19, especially among the global poor, cannot include forgoing vaccinations. But this caveat is not only for lower-income countries. Despite the overall high rate of coverage of essential child immunization, HICs are also insecure [55], as we attempt to highlight in our review. This high coverage grants protection for some VPDs attenuating the immediate effects of vaccine disruption, however even short gaps in vaccination coverage can result in outbreaks of VPDs. Postponement of campaigns comes at a cost. Vaccinations are time sensitive, and if children are not immunized within the correct age-window, they forego benefits of life long immunity [56]. Whole cohorts of children may be left unprotected.

Studies also demonstrated regional variation in the decline of vaccination coverage $[33,37]$. This variation could be due to regional cultural differences affecting levels of compliance with health guidelines, level of concern regarding infection, and differences in socioeconomic status. The schism in immunization coverage between different wealth quintiles and urban/rural residence has been previously established in literature $[57,58]$. Stakeholders must not forget the disparities in vaccination coverage and remember that much remains to be done if the benefit of childhood immunization is to be maximized.

COVID-19 is a magnifying glass that has highlighted the larger threat of two existing public health challenges, namely polio and measles. The long-term trajectory of the pandemic is uncertain, and the risk of resurgence of epidemic-prone diseases is very high. Additionally, at the same time the health system's capacity to perform a meaningful epidemiological analysis of the situation may also be impacted. Pakistan and Afghanistan were already struggling to eradicate polio [59], and with suspension of activities the risk of an epidemic is at the brink. In Pakistan, approximately 25,000 polio workers were diverted to help with the COVID-19 response [33]. Concerns of re-emergence of polio in previously polio-free countries escalated when Niger reported a new polio outbreak in April 2020 [60]. Discontinuation of polio vaccination programs may provide the virus a milieu to spread further and faster and could also result in worldwide export of infections. In addition, measles immunization campaigns have been suspended in 23 countries affecting almost 80 million eligible children [14]. Because of the highly infectious nature of measles, even 
a small decrease in routine measles vaccinations-a plausible result of lockdowns and disruption of health services-could lead to large and explosive outbreaks, which could raise the burden of childhood deaths significantly. It is imperative to ensure continuity of timely measles and polio vaccine administration for reaching the most vulnerable children and protecting them from common infectious disease. Policy makers must address the terrible calculus in determining how to proceed and capture the considerable loss of health from a wide range of diseases.

COVID-19 still presents as an evolving scenario with varying manifestations and emerging prevention and treatment strategies [61,62]. With this ever-evolving scenario, the pressure to meet spiraling health system needs is strong and a growing concern among virologists and public health experts, some wonder if the world has done more collateral damage in an attempt to curb the spread. Thus, while the drivers of low immunization coverage might lie outside of the health system, there are interventions that health policy makers could consider to mitigate disruptions and prioritize vaccination programs as avoiding vaccine-preventable deaths is critical for nations to preserve their gains in child survival.

The review has identified the following limitations. First, we identified a very limited number of studies on the impact of the COVID-19 pandemic on immunization coverage, particularly those that have reported the barriers or factors affecting the disruptions from users or providers perspective. Secondly, studies reported outcomes differently and therefore we could not pool or meta-analyze them. Third, the majority, but not all, compared the disruption of pandemic on vaccination coverage with pre-COVID vaccination coverage, and therefore we couldn't report the actual impact in numbers or proportions. However, as the pandemic continues, there are limited data and not all studies reported pre-pandemic numbers for comparison. As we attempt to highlight the impact of COVID19 on pre-existing immunization programs, factors such as the cost of vaccines and policies determining immunization have not been explored.

\section{Conclusions}

The COVID-19 pandemic has disrupted health and health systems worldwide, and most countries have still not recovered from the immediate effects of the increased mortality and morbidity due to SARS-CoV-2 infection. The global pandemic may affect the immunization program by leading to a decline in financial, human, and other resources, social-instability, limiting political attention and the country's health priorities. This, in addition to the devastating consequences of the prolonged lockdowns, will challenge both HICs and LMICs for years to come, irrespective of their health infrastructure. Additionally, declines in vaccination coverage and refusal to vaccinate during the pandemic may adversely impact the dissemination of an imminent SARS-CoV-2 vaccine. It is essential to urgently address the basic needs of health care facilities and resume routine health services with appropriate COVID-19 preventive measures in place. The need to define an integrated strategy that could involve all entities to safeguard individuals and mitigate the impact of the outbreak is crucial.

Supplementary Materials: The following are available online at https:/ / www.mdpi.com/1660-460 1/18/3/988/s1, Figure S1: PRISMA Checklist, Table S1: Search strategy.

Author Contributions: R.N. and F.S. screened the search results, screened the retrieved papers against the inclusion criteria, extracted the data and completed the data analysis. R.N., F.S., R.A.S. drafted the initial manuscript, reviewed and revised the manuscript. J.K.D., Z.S.L., R.A.S. designed the study, coordinated and supervised data collection, and critically reviewed and modified the manuscript. All authors approved the final manuscript as submitted and agree to be accountable for all aspects of the work. All authors have read and agreed to the published version of the manuscript.

Funding: This work was not supported by any funds.

Institutional Review Board Statement: Ethical review and approval were waived for this study as this was a systematic review. 
Informed Consent Statement: Not Applicable.

Data Availability Statement: All data is available upon reasonable request.

Conflicts of Interest: Authors have no conflict of interest.

\section{References}

1. WHO Director-General's Opening Remarks at the Media Briefing on COVID-19-11 March 2020. Available online: https:/ /www. who.int/dg/speeches / detail/who-director-general-s-opening-remarks-at-the-media-briefing-on-covid-19---11-march-2020 (accessed on 31 July 2020).

2. Atique, S.; Zarour, A.; Siddiqui, T.; El-Menyar, A.; Maull, K.; Al Thani, H.; Latifi, R. Trauma caused by falling objects at construction sites. J. Trauma Acute Care Surg. 2012, 73, 704-708. [CrossRef] [PubMed]

3. Oyo-Ita, A.; Wiysonge, C.S.; Oringanje, C.; Nwachukwu, C.E.; Oduwole, O.; Meremikwu, M.M. Interventions for improving coverage of childhood immunisation in low- and middle-income countries. Cochrane Database Syst. Rev. 2016, 7, Cd008145. [CrossRef] [PubMed]

4. PAHO. The Immunization Program in the Context of the COVID-19 Pandemic, 26 March 2020; PAHO: Washington, DC, USA, 2020.

5. WHO. Guiding Principles for Immunization Activities during the COVID-19 Pandemic. Interim Guidance. 26 March 2020. Available online: https:/ / apps.who.int/iris/bitstream/handle/10665/331590/WHO-2019-nCoV-immunization_services-2020 .1-eng.pdf (accessed on 31 July 2020).

6. WHO. Progress and Challenges with Achieving Universal Immunization Coverage. 2017 WHO/UNICEF Estimates of National Immunization Coverage [internet]; World Health Organization: Geneva, Switzerland, 2019.

7. WHO. At Least 80 Million Children under One at Risk of Diseases Such as Diphtheria, Measles and Polio as COVID-19 Disrupts Routine Vaccination Efforts, Warn GAVI, WHO and UNICEF. Available online: https://www.who.int/news-room/detail/22-052020-at-least-80-million-children-under-one-at-risk-of-diseases-such-as-diphtheria-measles-and-polio-as-covid-19-disruptsroutine-vaccination-efforts-warn-gavi-who-and-unicef (accessed on 5 August 2020).

8. Nelson, R. COVID-19 disrupts vaccine delivery. Lancet Infect. Dis. 2020, 20, 546. [CrossRef]

9. Zar, H.J.; Dawa, J.; Fischer, G.B.; Castro-Rodriguez, J.A. Challenges of COVID-19 in children in low- and middle-income countries. Paediatr. Respir. Rev. 2020, 35, 70-74. [CrossRef]

10. GAVI. COVID-19: Massive Impact on Lower-Income Countries Threatens More Disease Outbreaks. Available online: https: //www.gavi.org/news/media-room/covid-19-massive-impact-lower-income-countries-threatens-more-disease-outbreaks (accessed on 9 August 2020).

11. Hoffman, J.M.R. Slowing the Coronavirus is Speeding the Spread of Other Diseases. New York Times. Available online: https:/ / www.nytimes.com/2020/06/14/health/coronavirus-vaccines-measles.html (accessed on 5 August 2020).

12. This Week. Polio This Week as of 13 January 2020. Available online: http://polioeradication.org/polio-today/polio-now/thisweek/ (accessed on 5 August 2020).

13. Bagchi, S. COVID-19 and measles: Double trouble for Burundi. Lancet Microbe 2020, 1, e65. [CrossRef]

14. Roberts, L. Why measles deaths are surging- and coronavirus could make it worse. Nature 2020, 580, 446-447. [CrossRef] [PubMed]

15. Initiative, M.a.R. More than 117 Million Children at Risk of Missing Out on Measles Vaccine, as COVID-19 Surges. Available online: https:/ / measlesrubellainitiative.org/measles-news/more-than-117-million-children-at-risk-of-missing-out-on-measlesvaccines-as-covid-19-surges / (accessed on 31 July 2020).

16. Navarro, J.; Arrivillaga-Henríquez, J.; Salazar-Loor, J.; Rodriguez-Morales, A.J. COVID-19 and dengue, co-epidemics in Ecuador and other countries in Latin America: Pushing strained health care systems over the edge. Travel Med. Infect. Dis. 2020, $37,101656$. [CrossRef] [PubMed]

17. Nacher, M.; Douine, M.; Gaillet, M.; Flamand, C.; Rousset, D.; Rousseau, C.; Mahdaoui, C.; Carroll, S.; Valdes, A.; Passard, N.; et al. Simultaneous dengue and COVID-19 epidemics: Difficult days ahead? PLoS Negl. Trop. Dis. 2020, 14, e0008426. [CrossRef] [PubMed]

18. Adepoju, P. Lessons from Ebola as DRC grapples with conflict, measles, and covid-19. BMJ 2020, 370, m2879. [CrossRef] [PubMed]

19. Sun, X.; Samba, T.T.; Yao, J.; Yin, W.; Xiao, L.; Liu, F.; Liu, X.; Zhou, J.; Kou, Z.; Fan, H.; et al. Impact of the Ebola outbreak on routine immunization in western area, Sierra Leone-A field survey from an Ebola epidemic area. BMC Public Health 2017, 17, 363. [CrossRef]

20. Verity, R.; Okell, L.C.; Dorigatti, I.; Winskill, P.; Whittaker, C.; Imai, N.; Cuomo-Dannenburg, G.; Thompson, H.; Walker, P.G.T.; $\mathrm{Fu}, \mathrm{H}$;; et al. Estimates of the severity of coronavirus disease 2019: A model-based analysis. Lancet Infect Dis 2020, 20, 669-677. [CrossRef]

21. WHO. The Potential Impact of Health Service Disruptions on the Burden of Malaria: A Modelling Analysis for Countries in Sub-Saharan Africa; World Health Organization: Geneva, Switzerland, 2020.

22. Vitek, C.R.; Wharton, M. Diphtheria in the former Soviet Union: Reemergence of a pandemic disease. Emerg. Infect. Dis. 1998, 4, 539-550. [CrossRef] [PubMed] 
23. Moher, D.; Liberati, A.; Tetzlaff, J.; Altman, D.G. Preferred reporting items for systematic reviews and meta-analyses: The PRISMA statement. PLoS Med. 2009, 6, e1000097. [CrossRef] [PubMed]

24. Galvagno, S.M., Jr.; Massey, M.; Bouzat, P.; Vesselinov, R.; Levy, M.J.; Millin, M.G.; Stein, D.M.; Scalea, T.M.; Hirshon, J.M. Correlation between the revised trauma score and injury severity score: Implications for prehospital trauma triage. Prehospital Emerg. Care 2019, 23, 263-270. [CrossRef]

25. Grabenstein, J.D.; Nevin, R.L. Mass immunization programs: Principles and standards. Curr. Top Microbiol. Immunol. 2006, 304, 31-51. [CrossRef]

26. Covidence. Covidence Systematic Review Software; Covidence: Melbourne, VIC, Australia, 2017.

27. NHLBI. Study Quality Assessment Tools; NHLBI: Bethesda, MD, USA, 2019.

28. Haqqi, A.; Zahoor, S.; Aftab, M.N.; Tipu, I.; Rehman, Y.; Ahmed, H.; Afzal, M.S. COVID-19 in Pakistan: Impact on global polio eradication initiative. J. Med. Virol. 2021, 93, 141-143. [CrossRef]

29. Bechini, A.; Garamella, G.; Giammarco, B.; Zanella, B.; Flori, V.; Bonanni, P.; Boccalini, S. Paediatric activities and adherence to vaccinations during the COVID-19 epidemic period in Tuscany, Italy: A survey of paediatricians. J. Prev. Med. Hyg. 2020, 61, E125-E129. [CrossRef]

30. Bramer, C.A.; Kimmins, L.M.; Swanson, R.; Kuo, J.; Vranesich, P.; Jacques-Carroll, L.A.; Shen, A.K. Decline in Child Vaccination Coverage During the COVID-19 Pandemic-Michigan Care Improvement Registry, May 2016-May 2020. MMWR Morb. Mortal. Wkly. Rep. 2020, 69, 630-631. [CrossRef]

31. Buonsenso, D.; Cinicola, B.; Kallon, M.N.; Iodice, F. Child Healthcare and Immunizations in Sub-Saharan Africa During the COVID-19 Pandemic. Front. Pediatr. 2020, 8, 517. [CrossRef]

32. Chanchlani, N.; Buchanan, F.; Gill, P.J. Addressing the indirect effects of COVID-19 on the health of children and young people. CMAJ 2020, 192, E921-E927. [CrossRef]

33. Chandir, S.; Siddiqi, D.A.; Setayesh, H.; Khan, A.J. Impact of COVID-19 lockdown on routine immunisation in Karachi, Pakistan. Lancet Glob. Health 2020, 8, e1118-e1120. [CrossRef]

34. Chard, A.N.; Datta, S.D.; Tallis, G.; Burns, C.C.; Wassilak, S.G.F.; Vertefeuille, J.F.; Zaffran, M. Progress Toward Polio EradicationWorldwide, January 2018-March 2020. MMWR Morb. Mortal. Wkly. Rep. 2020, 69, 784-789. [CrossRef] [PubMed]

35. Kirmani, S.; Saleem, A. Impact of COVID-19 pandemic on paediatric services at a referral centre in Pakistan: Lessons from a low-income and middle-income country setting. Arch. Dis. Child. 2020. [CrossRef] [PubMed]

36. Langdon-Embry, M.; Papadouka, V.; Cheng, I.; Almashhadani, M.; Ternier, A.; Zucker, J.R. Notes from the Field: Rebound in Routine Childhood Vaccine Administration Following Decline During the COVID-19 Pandemic-New York City, March 1-June 27, 2020. MMWR Morb. Mortal. Wkly. Rep. 2020, 69, 999-1001. [CrossRef]

37. McDonald, H.I.; Tessier, E.; White, J.M.; Woodruff, M.; Knowles, C.; Bates, C.; Parry, J.; Walker, J.L.; Scott, J.A.; Smeeth, L.; et al. Early impact of the coronavirus disease (COVID-19) pandemic and physical distancing measures on routine childhood vaccinations in England, January to April 2020. Euro Surveill. 2020, 25. [CrossRef] [PubMed]

38. Moraga-Llop, F.A.; Fernández-Prada, M.; Grande-Tejada, A.M.; Martínez-Alcorta, L.I.; Moreno-Pérez, D.; Pérez-Martín, J.J. Recovering lost vaccine coverage due to COVID-19 pandemic. Vacunas 2020, 21, 129-135. [CrossRef]

39. Santoli, J.M.; Lindley, M.C.; DeSilva, M.B.; Kharbanda, E.O.; Daley, M.F.; Galloway, L.; Gee, J.; Glover, M.; Herring, B.; Kang, Y.; et al. Effects of the COVID-19 Pandemic on Routine Pediatric Vaccine Ordering and Administration-United States, 2020. MMWR Morb. Mortal. Wkly. Rep. 2020, 69, 591-593. [CrossRef] [PubMed]

40. Saxena, S.; Skirrow, H.; Bedford, H. Routine vaccination during covid-19 pandemic response. BMJ 2020, 369, m2392. [CrossRef]

41. Siedner, M.J.; Kraemer, J.D.; Meyer, M.J.; Harling, G.; Mngomezulu, T.; Gabela, P.; Dlamini, S.; Gareta, D.; Majozi, N.; Ngwenya, N.; et al. Access to primary healthcare during lockdown measures for COVID-19 in rural South Africa: A longitudinal cohort study. medRxiv 2020. [CrossRef]

42. Vogt, T.M.; Zhang, F.; Banks, M.; Black, C.; Arthur, B.; Kang, Y.; Lucas, P.; Lamont, B. Provision of Pediatric Immunization Services During the COVID-19 Pandemic: An Assessment of Capacity Among Pediatric Immunization Providers Participating in the Vaccines for Children Program-United States, May 2020. MMWR Morb. Mortal. Wkly. Rep. 2020, 69, 859-863. [CrossRef]

43. GAVI. COVID-19: Situation Report \#14. In COVID-19 Situation Reports; GAVI: Geneva, Switzerland, 2020.

44. Weill, A.; Drouin, J.; Desplas, D.; Cuenot, F.; Dray Spira, R.; Zureik, M. Usage des Médicaments de Ville en France Durant L'épidémie de la Covid-19-Point de Situation Après les 8 Semaines de Confinement et une Semaine de Post-Confinement (jusqu'au 17 mai 2020). Étude Pharmaco-Épidémiologique à Partir des Données de Remboursement du SNDS; GIS ANSM-Cnam: Saint-Denis, France, 2020; 312p.

45. The Lancet Child. Pandemic school closures: Risks and opportunities. Lancet Child. Adolesc. Health 2020, 4, 341. [CrossRef]

46. Huang, C.; Wang, Y.; Li, X.; Ren, L.; Zhao, J.; Hu, Y.; Zhang, L.; Fan, G.; Xu, J.; Gu, X.; et al. Clinical features of patients infected with 2019 novel coronavirus in Wuhan, China. Lancet 2020, 395, 497-506. [CrossRef]

47. Lee, P.I.; Hu, Y.L.; Chen, P.Y.; Huang, Y.C.; Hsueh, P.R. Are children less susceptible to COVID-19? J. Microbiol. Immunol. Infect. 2020, 53, 371-372. [CrossRef] [PubMed]

48. Roberton, T.; Carter, E.D.; Chou, V.B.; Stegmuller, A.R.; Jackson, B.D.; Tam, Y.; Sawadogo-Lewis, T.; Walker, N. Early estimates of the indirect effects of the COVID-19 pandemic on maternal and child mortality in low-income and middle-income countries: A modelling study. Lancet Glob. Health 2020, 8, e901-e908. [CrossRef] 
49. Macpherson, L.; Ogero, M.; Akech, S.; Aluvaala, J.; Gathara, D.; Irimu, G.; English, M.; Agweyu, A. Risk factors for death among children aged 5-14 years hospitalised with pneumonia: A retrospective cohort study in Kenya. BMJ Glob. Health 2019, 4, e001715. [CrossRef] [PubMed]

50. Clark, H.; Coll-Seck, A.M.; Banerjee, A.; Peterson, S.; Dalglish, S.L.; Ameratunga, S.; Balabanova, D.; Bhan, M.K.; Bhutta, Z.A.; Borrazzo, J.; et al. A future for the world's children? A WHO-UNICEF-Lancet Commission. Lancet 2020, 395, 605-658. [CrossRef]

51. Donohue, J.M.; Miller, E. COVID-19 and School Closures. JAMA 2020, 324, 845-847. [CrossRef]

52. Van Lancker, W.; Parolin, Z. COVID-19, school closures, and child poverty: A social crisis in the making. Lancet Public Health 2020, 5, e243-e244. [CrossRef]

53. Mills, A. Health Care Systems in Low- and Middle-Income Countries. N. Engl. J. Med. 2014, 370, 552-557. [CrossRef]

54. Abbas, K.; Procter, S.R.; van Zandvoort, K.; Clark, A.; Funk, S.; Mengistu, T.; Hogan, D.; Dansereau, E.; Jit, M.; Flasche, S. Routine childhood immunisation during the COVID-19 pandemic in Africa: A benefit-risk analysis of health benefits versus excess risk of SARS-CoV-2 infection. Lancet Glob. Health 2020, 8, e1267-e1272. [CrossRef]

55. Vigo, D.; Thornicroft, G.; Gureje, O. The Differential Outcomes of Coronavirus Disease 2019 in Low- and Middle-Income Countries vs High-Income Countries. JAMA Psychiatry 2020, 77, 1207-1208. [CrossRef] [PubMed]

56. Nic Lochlainn, L.M.; de Gier, B.; van der Maas, N.; Strebel, P.M.; Goodman, T.; van Binnendijk, R.S.; de Melker, H.E.; Hahné, S.J.M. Immunogenicity, effectiveness, and safety of measles vaccination in infants younger than 9 months: A systematic review and meta-analysis. Lancet Infect. Dis. 2019, 19, 1235-1245. [CrossRef]

57. Hosseinpoor, A.R.; Bergen, N.; Schlotheuber, A.; Gacic-Dobo, M.; Hansen, P.M.; Senouci, K.; Boerma, T.; Barros, A.J. State of inequality in diphtheria-tetanus-pertussis immunisation coverage in low-income and middle-income countries: A multicountry study of household health surveys. Lancet Glob. Health 2016, 4, e617-e626. [CrossRef]

58. Restrepo-Méndez, M.C.; Barros, A.J.; Wong, K.L.; Johnson, H.L.; Pariyo, G.; França, G.V.; Wehrmeister, F.C.; Victora, C.G. Inequalities in full immunization coverage: Trends in low- and middle-income countries. Bull. World Health Organ. 2016, 94, 794. [CrossRef]

59. G.P.E.I. Endemic Countries. Available online: http://polioeradication.org/where-we-work/polio-endemic-countries/ (accessed on 6 September 2020).

60. Niger Reports New Polio Outbreak. Available online: https://www.afro.who.int/news/niger-reports-new-polio-outbreak (accessed on 6 September 2020).

61. Singh, V.K.; Mishra, A.; Singh, S.; Kumar, P.; Singh, M.; Jagannath, C.; Khan, A. Emerging prevention and treatment strategies to control COVID-19. Pathogens 2020, 9, 501. [CrossRef] [PubMed]

62. Słomka, A.; Kowalewski, M.; Żekanowska, E. Coronavirus Disease 2019 (COVID-19): A Short Review on Hematological Manifestations. Pathogens 2020, 9, 493. [CrossRef] 